\title{
Der strategische Gebrauch der argumentativen Konjunktionen weil und denn in Fernsehdiskus- sionen bei Lerner- und MuttersprachlerInnen des Deutschen am Beispiel des Presseclubs
}

\author{
Andrea Jahnel
}

Dieser Beitrag stellt einen Teil einer Untersuchung ${ }^{1}$ dar, die der Frage nachgeht, inwiefern nicht muttersprachliche SprecherInnen, auch wenn sie fließend die Zielsprache sprechen, andere metakommunikative Strategien bzw. lernersprachliche Entsprechungen in einer für sie fremdsprachlichen Diskussion anwenden als muttersprachliche SprecherInnen. Als Untersuchungsgegenstand wählte ich »natürliche«, d. h. nicht inszenierte bzw. speziell für die Untersuchung elizitierte Gespräche. Der empirischen Analyse lag also authentisches Beispielmaterial $^{2}$ zugrunde, und zwar 10 Folgen des Presseclubs, einer Fernsehdiskussionsrunde mit ausländischen und deutschen JournalistInnen, die ein jeweils aktuelles politisches Thema diskutieren.

Methodisch wurden in der Untersuchung Ansätze der Text- und Gesprächs- analyse, der Medientheorie und der Sprachlehrforschung verbunden. Weiterhin wurde die ethnomethodologische Gesprächsanalyse in ihrer als Diskursanalyse weiterentwickelten Form, die Konversationsanalyse und sprechakttheoretische Argumentationstheorien verbindet, von mir angewendet.

Neben der Gesprächsorganisation, d.h. den formalen Strukturen der Gesprächsabläufe, die zum Themen- und Floormanagement gezählt werden, untersuchte ich diskussionstypische sprachliche Modalisierungsmöglichkeiten wie Modalpartikeln, interne und externe Indikatoren zur Markierung von Fragen, konzessive Adverbien, abschwächende und verstärkende Geltungs- und Intensitätsadverbien, Nexus- und Gradadverbien sowie das Positionsadverb $\mathrm{da}$, lexikalische Mittel der Abwertung, des Bedauerns und Entschuldigens, den Konjunk-

1 Die wesentlichen Teile der Untersuchung erscheinen als Dissertation voraussichtlich 1999 (Arbeitstitel: »Argumentation in der internationalen Diskussion«).

2 Die folgenden Zitate aus dem Korpus erhalten eine Signatur, in der das Datum des Gesprächs (z. B. 05.01.92), der Zeitpunkt der Äußerung im Gespräch in Minuten und Sekunden (z. B. 10:58) sowie das Kürzel der SprecherIn (z. B. Ba) enthalten ist. Die Zitate der MuttersprachlerInnen (M) und FremdsprachlerInnen (F) werden durchnumeriert. Die Konjunktionen weil und denn werden unterstrichen, sprachliche Elemente, auf die im Haupttext eingegangen wurde, fett gedruckt. Außerdem werden im Text die Abkürzungen $\mathrm{N}$ für muttersprachliche und $\mathrm{NN}$ für nicht muttersprachliche SprecherInnen gebraucht. 
tiv II und Modalverben, Heckenausdrükke, Verba sentiendi und sciendi, Anreden und syntaktische Mittel.

In diesem Beitrag soll nun der Gebrauch der argumentativen Konjunktionen weil und denn bei Lerner- und MuttersprachlerInnen des Deutschen verglichen werden.

Kausalgefüge, die durch entsprechende Konjunktionen realisiert werden, besitzen bestimmte Satz- und Strukturmuster, mit denen mögliche Argumente in eine Form gesetzt werden können. Kausale Konjunktionen zeigen an, daß die Bezugsäußerung eine »Behauptung « ist, ein strittiger Sachverhalt, der begründet werden soll. Diese Begründungskonjunktionen dienen der SprecherIn zur Sicherung der Gültigkeit ihres eigenen Beitrags und damit gegebenenfalls auch zur Zurückweisung des Anspruchs eines Opponenten oder einer Opponentin. Sie sind illokutive Indikatoren für eine Argumentation, beweisen aber noch nicht, daß die Sprechaktfolge wirklich eine Argumentation ist.

Das ka u sale weil leitet einen untergeordneten Nebensatz ein, so daß ein Teilsatz im abhängigen Skopus des anderen liegt, und eröffnet - im Gegensatz zum epistemischen Gebrauch von weil (s.u.) eine Klammer mit dem Verb in der Endstellung als klammerschließendem Element. Es bezieht sich auf den kausalen Ursache-Wirkungszusammenhang zwischen zwei Sachverhalten und ist ein Operator, der nicht einstellungskundgebend ist, sondern sich direkt auf Propositionen bezieht.

Das epistemische weil mit Verbzweitstellung realisiert pragmatische Phänomene (vgl. Günthner 1993), hat eine andere Diskursfunktion als das kausale weil und tritt in einer anderen sequentiellen Umgebung auf. ${ }^{1}$ Diese Verwendungsweise von weil, die von C. Küper (1989: 491) als diagnostische bezeichnet wird, kennzeichnet eine epistemische Erklärung, welche nicht durch einen Präpositionalausdruck wie auf Grund oder wegen substituiert werden kann. Mit dem weil-Satz wird keine Begründung für den Sachverhalt der Bezugsäußerung gegeben. Es handelt sich vielmehr beim epistemischen weil um einen sich auf die Sprechhandlung selbst beziehenden weil-Satz, um eine Sprechaktqualifikation, die sich auf Einstellungen zu Propositionen bezieht, und signalisiert, warum eine Person das Argument des Vordersatzes anführt, d.h. um eine Rechtfertigung dafür, daß dieser bestimmte Punkt angebracht wird. Der weil-Satz bietet dabei häufig keine wirklich neue Information. Es besteht keine enge Zusammengehörigkeit zwischen den beiden Teilsätzen: Sie sind getrennt assertierbar, können auch separat geäuBert werden und sind grammatikalisch voneinander unabhängig. Die lockere Beziehung zwischen den beiden Teilsätzen wird auch daran deutlich, daß man vor dem weil-Satz eine längere Sprechpause machen kann.

Die häufig in weil-Konstruktionen mit Verbzweitstellung auftretende Besetzung des Vorfeldes und die Vorausstellung eines Elements sind main clause phenomena (Günthner 1993), wodurch eine Linksversetzung und folglich eine Fokussierung erreicht wird. ${ }^{2}$ D.h., die Verbzweitstellung ist auch durch den Versuch bedingt, mittels einer Vorfeldbe-

1 Es kann also nach Günthner (1993) nicht von einer »Tendenz zur Hauptsatzstellung in deutschen Nebensätzen« gesprochen werden.

2 Zum Beispiel: (...) Und unser Ruf im Ausland wird sich natürlich immer mehr verschlechtern, weil uns Deutschen nimmt man das übel, obwohl die Generation jetzt... (08.11.92; 28:33; Rot). 
setzung eine bestimmte Betonung zu erreichen.

Das epistemische weil und die damit verbundene Verbzweitstellung können auch als floor-keeping-device ${ }^{1}$ (vgl. ebda.) interpretiert werden. So entsteht nach dem weil oft eine Pause oder eine Zögerungspartikel wird eingeschoben, da eigentlich keine wirkliche Kausalbeziehung intendiert war.

Die koordinierend-nebenordnende Konjunktion denn in Nullstellenposition wird gebraucht, um einen vorausgegangenen Satz mit einem vollständigen Hauptsatz zu verknüpfen. Deshalb kann man schwer entscheiden, ob man diese Sätze durch einen Punkt oder ein Komma voneinander trennen soll. Denn gibt eine nachgeschobene, für die SprecherIn in der gegebenen Situation bedeutsame, relativ ausführliche oder in einem informativ unauffälligen Kontext auffällige und weitestgehend eigenständige »Begründung für einen schwer verstehbaren Sachverhalt« (Weinrich 1993: 760). Gerade die syntaktische Selbständigkeit des denn-Adjunktes ermöglicht diese längere und eingehende Erklärung.

Denn ist als Indikator für sprechaktbezogene, epistemische Begründungen von Sprechhandlungen (Günthner 1993) ein sprachliches Mittel zur Herstellung von Verstehen, zwingt aber im Gegensatz zum kausalen weil den AdressatInnen die Meinung der SprecherIn nicht als schlüssige auf (Redder 1990). Günthner betrachtet die Konjunktion denn als »Stilvariante« des epistemischen weil, wobei denn in »spontanen Alltagsgesprächen weniger beliebt « und eher ein »Kennzeichen des >offiziellen« Sprachgebrauchs« sei.

Mit denn ist aufgrund seiner einstellungskundgebenden Funktion ein stärkerer interaktiv-sequentieller HörerInnenbezug gegeben, wodurch die SprecherIn einem möglichen Nichtverstehen seitens der HörerIn präventiv vorgreift. Denn-Sätze sind Behauptungen, wobei die SprecherIn sich ausdrücklich nur auf ihre eigenen Einschätzungs- und Bewertungsindikatoren bezieht.

Folgende Gebrauchsweisen von denn und weil traten bei $\mathrm{N}$ im Korpus besonders häufig auf.

a) Bei der Kausalkonjunktion weil wird der Begründungssatz von den nativen SprecherInnen oft zusätzlich betont, unterstützt und markiert, und zwar häufig durch ein vorausgehendes Nexusadverb (deshalb, darum, daher, deswegen) wie in den Beispielen M1 und M9, manchmal aber auch durch ein nachträgliches $\mathrm{Ne}-$ xusadverb wie in M8, durch Doppelpunktkonstruktionen (M3, M5 und M7) oder dadurch, daß man den begründenden weil-Teil an den Anfang des Satzes mit Initialstellung der Konjunktion schiebt (wie in den Beispielen M2 und M6). ${ }^{2}$ Auch das kausale weil selbst kann besonders betont werden, z. B. durch ein eingefügtes Gradadverb (M4). So wird signalisiert, daß der angeschlossene Grund sehr bedeutsam ist und in besonders betontem kausalen Zusammenhang zum Hauptsatz steht:

1 Ein floor-keeping-device ist ein konversationelles Fortsetzungssignal mit koordinierender Funktion, das den RezipientInnen deutlich macht, daß die aktuelle SprecherIn ihr Rederecht an einem transition-relevant-place, d.h. an einem geeigneten Platz zur Gesprächsschrittübergabe, nicht aufgeben will.

2 Dies tritt auch einmal bei einem NN auf, wobei die Betonung aber nicht auf weil, sondern auf nicht liegt: (...) Und weil man das nicht getan hat, es tut mir leid, ich komme immer zurück, dann ist man... (10.05.92; 36:33; Ca). 
M1 (...) Und zwar deswegen, weil sie eben auch graduell nach dem, nach... (05.01.92; 10:58; Ba)

M2 (...) Ich kann aber nur immer wieder sagen: Weil wir diese Problematik kennen und wissen, daß wir das... (12.01.92; 40:23; Ut)

M3 (...) Das hat aber einen Grund: Weil wir in Bayern durchgreifen, und dieses... (08.11.92; 14:11; Sch)

M4 (...), eben weil wir kein integratives System haben. Das heißt, daß... (08.11.92; 20:29; Da)

M5 (...) Es ist genau der Grund: Weil man da sehr Angst hat: Sobald es nämlich wirklich um Einwanderungsgesetze geht, müßte man... (08.11.92; 20:40; Da)

M6 (...) Weil man weiß, die Kamera ist da, wird schnell die Nazifahne entrollt, die man... (08.11.92; 42:26; Sch)

M7 (...) Ich glaube aus einem entscheidenden Grund: Weil wir uns immer wieder davor gedrückt haben zu sagen, notfalls... (22.11.92; 09:14; Be)

M8 Nein, die Menschen kommen zu uns, weil wir das reichste Land sind. Deswegen kommen die Menschen. Wenn... (06.12.92; 16:31; Fo)

M9 (...) Die kommen auch deswegen nicht mehr, weil wir strikte und rigide Veränderungspolitik betrieben. Die können... (06.12.92; 17:34; Pr)

b) Das eindeutig kausale weil wird von den $\mathrm{N}$ im Vergleich zu den non-nativen SprecherInnen eher bei einer parenthetischen Begründung benutzt. Der weil-Teilsatz bezieht sich also nur auf einen unwichtigeren bzw. unbedeutenderen Subbereich des im vorausgehenden Teilsatz geäußerten Sachverhalts, der theoretisch auch weggelassen werden könnte. Der Kausalsatz wird dabei manchmal nur als ein Einschub, z. B. in einem Konditionalgefüge, konstruiert, wobei die Verbendstellung unbedingt erforderlich ist:
M10 (...), daß wir jetzt nicht das Kind mit dem Bade ausschütten und wirklich 'ne isolierte Gruppe schaffen, die nur noch in diesem Parlament ist und da auch bleiben muß, weil sie in den Beruf nicht zurückkehren kann, so daß... (14.06.92; 18:05; Sü)

M11 (...) Etwas was man gerade hier, weil wir in einer journalistischen Runde sitzen und in einer Fernsehrunde, vielleicht auch einmal fragen sollte, das... (08.11.92; 42:04; Sch)

c) Ein kurzer einleitender, epistemischer ${ }^{1}$ weil-Satz, der eine Subjektivierung enthält, wird von $\mathrm{N}$ zuweilen durch eine Doppelpunktkonstruktion von einem relativ komplexen Satz abgetrennt, der wiederum aus einem Haupt- und einem Nebensatz besteht. Die epistemische Konstruktion offeriert die Möglichkeit, einen längeren Redebeitrag als Begründung nachzuliefern. In den Beispielen M13 und M14 enthält schon die Proposition des Vorsatzes ein Element, welches die Aussage als Meinung markiert (finde ich, ich persönlich) und an welches eine subjektive Begründung angeschlossen wird:

M12 (...) Sorgen für die Europäer ganz sicher, weil ich glaube: Wenn man das einmal historisch sieht, ist das Drama der Geschichte des 20. Jahrhunderts an uns Europäer viel näher herangerückt worden durch... (29.12.91; 04:40; St)

M13 (...) Das finde ich schon bedenklich. Nicht weil sie dort viel Geld verdienen, sondern weil ich denke: Wenn sie Abgeordnete sind, sind sie Abgeordnete. Das ist... (14.06.92; 16:25; Si)

M14 (...) Ich persönlich bin gegen diese Vorruhestandsregelung, auch in den neuen Ländern, weil ich denke: Wir kommen immer mehr dahin, inzwischen mit/die Leute mit 40... (14.06.92; 35:20; Sü)

1 Die Epistemizität kann hier nicht anhand der Verbstellung entschieden werden, sondern nur daran erkannt werden, daß für das kausale weil eine Verbindung mit einer Subjektivierung untypisch ist. 
d) Die epistemische Funktion von denn und die ausführliche Begründung, die durch denn eingeleitet wird, machen oft - wie in Beispiel M16 - eine Konstruktion notwendig, bei der zwischen denn und das durch einen Doppelpunkt abgetrennte Adjunkt ein Einleitungssatz geschoben wird. Auffällig ist, daß das denn-Adjunkt der $\mathrm{N}$ - ähnlich wie der weil-Satz der NN (s.u.) - eher lang ist und in fast $1 / 3$ aller Fälle, aber in keinem einzigen Fall der NN, selber noch einmal eine wenn-Junktion umfaßt (vgl. M15, M17 und M18), so daß man nach den deutschen Interpunktionsregeln vor das denn auch einen Punkt setzen könnte. Die wenn-dann-Konstruktion ist formal und strukturell selbständig sowie aussagemäßig abgeschlossen. Denn hat hier eine Scharnierfunktion und ist ein Signal für Redefortsetzung. Ein ähnliches Phänomen zeigt sich bei anderen Anschlüssen, die eine Nebensatzkonstruktion einschließen:

M15 (...) Denn, und da nehm ich eine ganz einfache Sache, wenn - Sie haben das als Verteilungssystem (...) bezeichnet, und da... (05.01.92; 06:16; Le)

M16 (...) Denn in der Tat ist es so: Da ist ein Zusammenbruch der Wirtschaft, und wir... (12.01.92; 24:22; Neu)

M17 (...) $\ddot{A} h$ es gibt einige, auch mit guten Pfründen besetzt, denn also, wenn eben die Aufsichtsräte oder andere Gremien, in denen nicht... (14.06.92; 40:45; Sü)
M18 (...) Ich fürchte, daß äh Hans Apel recht hat, äh denn, wenn man ' $n$ bißchen die $S P D$ von innen kennt, dann glaube ich, daß das äh vor allen Dingen mit seinem Verhalten... (14.06.92; 42:22; Ka)

Das Vorkommen expliziter Kausalbeziehungen mit Konjunktionen ist in den 10 analysierten Sendungen des Presseclubs bei $\mathrm{N}$ und $\mathrm{NN}$ etwa gleich häufig $(85 \mathrm{mal}$ bei $\mathrm{N}$ und $83 \mathrm{mal}$ bei $\mathrm{NN}$ ). $\mathrm{N}$ verwenden 62mal weil, NN 73mal. N gebrauchen jedoch mehr als doppelt so häufig denn als NN (23mal bei N im Vergleich zu $10 \mathrm{mal}$ bei NN). In Verbendstellung wird weil von den $\mathrm{N} 47 \mathrm{mal}$, von den $\mathrm{NN}$ 38mal gebraucht. In Verbzweitstellung kommt es $7 \mathrm{mal}$ bei $\mathrm{N}$, jedoch $27 \mathrm{mal}$ bei NN vor. ${ }^{1}$

Bei der von NN im Gegensatz zu N wesentlich häufigeren Verwendung eines weil-Satzes mit Verbzweitstellung kann es sich um ein grammatikalisches Problem handeln. ${ }^{2}$ Das weil kann aber auch als epistemische Verknüpfung in der denn-Funktion gebraucht worden sein. Da in den meisten Fällen jedoch das weil durch ein denn substituiert werden kann, ist auf die Verwendung des epistemischen weil zu schließen. Gerade an den Beispielen F1 und F5 sieht man, daß ein epistemisches weil nicht die Proposition des Vorsatzes selbst begründet, sondern die Tatsache, warum die SprecherIn die vorangegangene Aussage gemacht hat. Hierauf deutet auch der offensichtliche

1 In den restlichen Fällen - je 8 für N und NN - kann man diese Differenzierung nicht vornehmen, da die Verbzweitstellung gleichzeitig die Verbendstellung ist (vgl. die Beispiele M12, M13 und M14). Man darf außerdem nicht voreilig zu dem Schluß kommen, daß es sich bei der Verbendstellung immer um ein kausales und bei der Verbzweitstellung stets um ein epistemisches weil handelt, da eine solche Zuordnung gerade bei NN - aufgrund von grammatikalischen Wortstellungsfehlern nicht zulässig ist.

2 Durch die Angabe eines Grundes auf eine warum-Frage in folgendem Beispiel ist klar, daß es sich um ein kausales weil handelt. Dennoch steht das finite Verb nicht in Endstellung: (...) Warum ist man in diese Lage gekommen? Im Grunde genommen weil vor einem/einem Jahr man hat nicht die ganze Herausforderung der deutschen Einheit dieser Nation gezeigt. Ich... (10.05.92; 18:13; Ca). 
Neuansatz nach einer Schlußfolgerung mit also (F4) und dann (F5 und F6) hin. Dabei wird zuweilen mit dem weil-Satz eine längere, aus mehreren Sätzen bestehende Begründungskette initiiert (wie in Beispiel F6).

Auch die Verbindung von Teilsätzen mit unterschiedlicher illokutionärer Funktion wie der Verknüpfung eines Interrogativ- und eines weil-Satzes, die auf deren relative Unabhängigkeit verweist $(\mathrm{F} 1$ und F8), läßt auf den epistemischen Gebrauch von weil schließen. Diese ist $\mathrm{u}$. a. auch an der Intonationskontur erkennbar, da zwischen dem weil und der folgenden Phrase eine Pause entsteht. Die vorangegangene Äußerung hat eine finale Tonhöhenbewegung. Der Fokus der Äußerung liegt auf der Kausalverknüpfung, welche einen eigenen Akzent trägt. Häufig knüpfen die NN, als Komplement zu den denn-wenn-Konstruktionen der $\mathrm{N}$, ein Konditionalgefüge an die Konjunktion weil an, wie in den Beispielen F1, F3, F7 und F8. Der erste Teilsatz stellt bei den NN oft - wie in den Beispielen F2 und F3 - nur ein Widerspruchssignal dar, wobei der Widerspruch nach dem epistemischen weil näher expliziert wird:

F1 (...) Ich habe überall gefragt: > Wie sieht es jetzt/jetzt aus mit der Neuordnung des UNO-Sicherheitsrats?< Äh weil: Wenn man davon ausgeht, daß die Amerikaner nicht mehr alleine führen können, und man sucht sich ein Gremium aus, was das macht, dann könnte man an den UNOSicherheitsrat denken. Das sind... $(10.05 .92 ; 31: 51 ; \mathrm{Kn})$

F2 (...) Das genügt nicht, weil: Die Frage ist, ob man/ob dieser/dieser Planet verkraftet, daß... (31.05.92; 03:51; Al)

F3 Ich möchte zuerst zu der Bevölkerung sagen. Ich finde, das ist immer eine $A b-$ lenkung, weil: Oft, wenn drüber gesprochen wird, was kann gemacht werden in unseren Ländern, sagt man, wir sind $z u$ viele. Das... (31.05.92; 08:38; Ob)
F4 (...) Also von der Bevölkerungs/üb/explosion kann nicht die Rede sein, weil: Platz haben wir genug. (31.05.92; 09:13; Ob)

F5 (...) Und dann können sich auch diese Probleme lösen. Natürlicherweise. Weil: Man stellt immer die Bevölkerung, diese Überbevölkerung im Vordergrund, und ich finde, dieses Problem... (31.05.92; 09:33; $\mathrm{Ob})$

F6 (...) Und wenn die aber sagen, das ist nicht mehr gut und etwas anderes ist gut, dann werden wir umsteigen, man/weil: Man sieht das auch jetzt in Rio. Niemand ist einer Meinung dort. Und die Länder des Südens haben sich zusammengetan und sind kräftig nicht einer Meinung. Und... (31.05.92; 23:27; Ob)

F7 (...) Sie müssen das zugeben, daß der Urwald äh geschützt werden muß, weil: Wenn es/wenn man äh warten will, daß in 20 Jahren jeder gut leben wird, dann ist zu spät/ist zu spät. Das ist... (31.05.92; 27:58; Ma-Rü)

F8 (...) Und wa/warum? Weil: Wenn man die äh Konzeption der Menschenrechte je/äh sch/äh zum Beispiel Frankreich oder/oder in/äh in Deutschland, ist da/ist/ gibt es äh Meinungsverschiedenheiten. Zum Beispiel... (08.11.92; 30:35; Ros)

Während die $\mathrm{N}$ an das denn oft komplexe Sätze anschließen, gebrauchen NN diese Konjunktion auch in sehr kurzen nachgeschobenen Begründungen:

F9 (...) Und es geht äh immer noch an alauch äh eh für die Armee um die soziale Absicherung, denn viele Soldaten sind unzufrieden. Äh wenn... (05.01.92; 40:17; Bo)

F10 (...) Aber jetzt Priorität Lebensmittel bitte, denn sie brauchen es dringend. Ich kann auch... (12.01.92; 39:19; Fi-Ru)

Wahrscheinlich ist der häufige Gebrauch von weil durch die NN darauf zurückzuführen, daß weil im Prozeß des Fremdspracherwerbs ontogenetisch früher erworben wird. Es gehört zu den ökonomischen Strategien von LernerInnen, ein Element einer Funktionsklasse besonders häufig zu benutzen, bevor man subtilere Unterscheidungen vornimmt. 
Da ein epistemisches weil eine ähnlich argumentative Funktion hat wie andere Modalitätsmarkierungen, z. B. die Modalpartikeln $j a$, doch, auch und eben ${ }^{1}$, die von NN extrem selten gebraucht werden, könnte man die These wagen, daß NN zwei Sätze eher durch die im Zweit- und Fremdspracherwerb leichter zu erlernende und für den mündlichen, alltagssprachlichen Gebrauch typische Konjunktion weil verknüpfen und damit ihren Text organisieren.

Der häufigere Gebrauch von denn durch $\mathrm{N}$ kann darauf zurückzuführen sein, daß Sätze mit denn nur in Passagen mit argumentativen Textstrukturen zulässig sind und daß Begründungen mit denn in Diskussionen überwiegen, in denen Wissen von »ExpertInnen « gefragt ist, z. B. in Streitgesprächen vor einem Fernsehpublikum. $^{2}$

Die Analyse des Gebrauchs der argumentativen Konjunktionen weil und denn festigt die auch für die anderen Analysegegenstände aufgestellte These, daß die für den Kontext Fernsehdiskussion geltenden, stillschweigend vorausgesetzten Regeln, Konventionen und rhetorisch-sti- listischen Strategien der dominierenden und durchsetzungsstärkeren Gastgeberkultur, die von den non-nativen TeilnehmerInnen nicht vollständig internalisiert wurden, zu einer Machtasymmetrie unter dem Deckmantel der oberflächlichen »Gleichheit« führen.

Insofern könnte man die sprachlich-stilistischen Mittel der non-nativen SprecherInnen auch als »machtlose « Sprache bezeichnen, die zwar einerseits eine Atmosphäre der Übereinstimmung und Harmonie herstellt und damit eine Gruppenstabilisierung erzeugt, aber andererseits dadurch, daß Sprache immer auch ein Mittel der impliziten Selbstdarstellung und der sozialen Identifikation ist, die Überzeugungskraft der Argumente schwächt und folglich erhebliche Konsequenzen für das Image der SprecherInnen hat.

Daraus leitet sich die Forderung für die Sprachlehrforschung ab, mit Einbeziehung konversationsanalytischer Ansätze dazu beizutragen, SprachlernerInnen für reales Sprachhandeln im fremdkulturellen Kontext zu sensibilisieren. Dies würde nicht nur zu einer Verbesserung der

1 Da man z. B. in Begründungen oft Evidenzmarkierungen benutzt, könnte eine Explikation der begründenden Relation zwischen zwei Behauptungen durch den Gebrauch einer Kausalkonjunktion ein Ersatz für eine $j a$-Äußerung sein. Ebenso werden die Modalpartikeln auch und eben häufig gebraucht, um kausale Zusammenhänge zu verdeutlichen. Natürlich haben die Kausaljunktionen und die Modalpartikeln keine vollkommen identischen Funktionen, denn sonst wären die häufig vorkommenden Äußerungen, die beide Elemente enthalten, redundant. Da die Modalpartikel ja oft in emotional geladenen Situationen (z. B. in Ausrufen) vorkommt, die das Eingreifen der SprecherIn fordern, läßt sich $j a$ nicht immer durch eine kausale Konjunktion ersetzen. Während die kausalen Konjunktionen nur eine Begründungsfunktion haben, haben die Modalpartikeln $a u c h, j a$ und eben vielfältige Funktionen. Zum Beispiel hat die Modalpartikel ja neben der Begründungsfunktion auch die Aufgabe, durch den Hinweis auf die Evidenz an die AdressatInnen den Appell zu richten, die Äußerung als Begründung auch anzunehmen (vgl. Jahnel demn.).

2 Parallel zu diesen Einzelergebnissen ließen sich auch die Resultate der übrigen Teile der Untersuchung in Gegensatzpaare bündeln, die das native und non-native Sprachverhalten plakativ und kontrastierend beschreiben: Markierung vs. Vagheit, Verstärkung vs. Abschwächung, aktive Initiative vs. passive Reaktion, Dialogizität vs. Monologizität, Kohärenz und logische Stringenz vs. unklare Zusammenhänge, Prägnanz vs. Elaboration sowie Aggression vs. Harmonie (vgl. Jahnel demn.). 
Argumentationsfähigkeit in der Zielsprache führen, sondern auch zu einem besseren Verständnis der eigenen muttersprachlichen Argumentationsstrategien, indem man diese aus der Fremdperspektive betrachtet.

\section{Literatur}

Günthner, Susanne: »...weil - man kann es ja wissenschaftlich untersuchen " - Diskurspragmatische Aspekte der Wortstellung in WEIL-Sätzen ", Linguistische Berichte, H. 143 (Jahrgang 1993/2), 37-59.
Jahnel, Andrea: »Ich glaube, ich würde gern auch $z u$ diesem Vorbild was sagen «. LernerInnen- und muttersprachliche Verwendung von Modalitätsstrategien in Fernsehdiskussionen am Beispiel des Presseclubs. (erscheint demnächst).

Küper, Claudia: »Kausale Satzverknüpfer«. In: Weydt, Harald (Hrsg.): Sprechen mit Partikeln. Berlin; New York 1989, 488-497.

Redder, Angelika: Grammatiktheorie und sprachliches Handeln: »denn « und »da«. Tübingen 1990.

Weinrich, Harald: Textgrammatik der deutschen Sprache. Mannheim 1993.

Weydt, Harald (Hrsg.): Sprechen mit Partikeln. Berlin; New York 1989. 